\title{
EVALUATION OF OPERATIONAL POLICIES IN THE DESIGN PHASE OF MATERIAL HANDLING NETWORKS
}

\author{
Ardavan Asef-Vaziri \\ Systems and Operations Management \\ College of Business and Economics \\ California State University, Northridge \\ 18111 Nordhoff St. \\ Northridge, CA 91330-8378, USA
}

\begin{abstract}
We compare quality of solution and solution times of alternative operational dispatching policies that are integrated into the design phase of a material handling network for automated guided vehicle systems. Exact formulations describe the problem of optimal concurrent design of the unidirectional loop track layout along with the locations of the pickup and drop-off stations. The objective is to minimize the total loaded and empty vehicle trip distances, which is the main driver of the fleet size of the vehicles. A primary interest of our work is to illustrate the superiority of the shortest-trip-distance-first (STDF) over the first-come-first-served (FCFS) when integrated into the design phase. We also show that optimization under STDF in the design phase produces the best solution for first-encountered-first-served (FEFS) in the operation phase. The findings of the optimization models in the design phase are well supported by the outcomes of the simulation model in the operation phase.
\end{abstract}

\section{INTRODUCTION}

One of the main tasks in designing efficient and economically viable automated material handling systems is the concurrent determination of the layout of the vehicle guide path tracks and the location of the pickup (P) and drop-off (D) stations. Each work-center (e.g. a department, a cell, a station) in a block layout is shown by a right angle polygon, which is not necessarily convex. The block layout of an 11-work-center example taken from Sinriech and Tanchoco (1993) and its required loaded flow matrix is shown in Figure 1. Elements of the loaded flow matrix correspond to the number of unit loads moved from the P station of the origin work-center in the row to the $\mathrm{D}$ station of the destination work-center in the column over a specific time horizon.
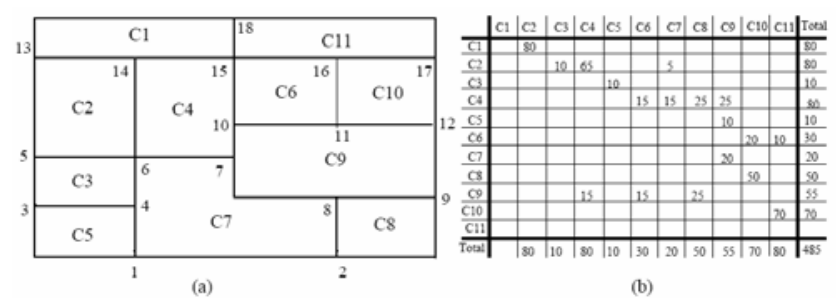

Figure 1: The 11-work-center prototype problem; (a) the block layout and (b) the loaded flow matrix.

Edges in a block layout defining the boundaries of the work-centers are considered as candidates to form the material handling network. Each work-center requires one $\mathrm{P}$ and one D station which are not necessarily combined. Nodes that are defined by the edges' intersections are candidates for station locations. The handling devices used in this study are unit load automated material handling vehicles. According to the Material Handling Industry of America, unit load carriers represent the single largest segment of automated material handling vehicles.

Unidirectional flow paths are easier to control and less expensive to implement. A conventional configuration for automated guided vehicle (AGV) systems is a unidirectional network formed by all the edges on the block layout. A unidirectional loop covering at least one edge of each work-center occupies a smaller portion of the block layout than conventional configurations. It also simplifies traffic management, vehicle routing, and dispatching decisions since a unidirectional loop has no intersection. However, longer trip distances and more blocking may be observed on a unidirectional loop.

In this study we analyze the efficiency (solution time) and effectiveness (quality of the solution) of the three dispatching policies of FCFS, STDF, and FEFS when integrated into the concurrent design of the loop and station locations. The objective function is the minimization of total loaded and empty vehicle trip distances which is the 
main driver of the fleet size of the vehicles. Our computations show that dictating the FCFS empty trips as input data to the optimization model leads to over-estimation of the fleet size of the vehicles and sub-optimization of the design. The model integrated with STDF empty trips outperforms the FCFS model.

The interest of this conclusion is in supporting STDF which is deterministic in the design phase, against FCFS which has been promoted due to its stochastic appearance. Furthermore, since FEFS can not be implemented on a loop and station locations which have not been designed yet, we argue that the best loop and stations for FEFS in the operations phase are recommended by STDF in the design phase. The analytical results are well supported by simulation experiments. The stochastic results of FEFS in the operation phase are very close to the deterministic estimates made by STDF in the design phase.

\section{NARRATIVE REPRESENTATION OF THE MODELS}

The optimization model for the simultaneous design of the loop and station locations is described as follows.

1. Constraint at-least-one-edge requires each workcenter to have at least one edge on the loop.

2. Constraint loop-based-configuration enforces each node to have either zero or two edges on the flow path.

3. Constraint no-sub-tour does not allow formation of disjoint loops.

4. Constraints unidirectional-loop assigns direction to an undirected loop.

5. Constraints one-P-one-D-station requires each work-center to have one $\mathrm{P}$ and one $\mathrm{D}$ station on the loop.

6. Constraint loaded-flow-feasibility states that the loaded vehicles can move only on the set of arcs that are selected to form the loop.

7. Constraint loaded-flow-conservation is the flow conservation constraint on the loaded vehicles.

Under the FCFS dispatching and its variations, the volumes of empty trips are parameters and are considered input data to the optimization model. The objective function of the total loaded and FCFS empty trip distances is defined based on the loaded and empty trip data given to the model, and locations and distances identified by the optimization model. The following constraints are required under FCFS dispatching.

8. Constraint FCFS-flow-feasibility is the same as Constraint (6) except for the empty trips.
9. Constraint FCFS-flow-conservation is similar to Constraint (7), except that, flows initiate from D stations and emanate at $\mathrm{P}$ stations.

Under the STDF dispatching and its variations, the empty vehicle trips are not parameters; they are decision variables to be optimized in their relation to loaded trips. The objective function of the total loaded and STDF empty trip distances is defined based on the loaded trip data given to the model, and locations, distances and empty trips identified by the optimization model. The following constraints are required under FCFS dispatching.

10. Constraint STDF-flow-feasibility states that empty vehicles can move only on the set of arcs that exist on the loop.

11. Constraint loaded-and-STDF-flow-conservation state that the total loaded and STDF empty vehicles entering and leaving a node are equal.

\section{SIMULATION}

In the prototype example of Section 1 we can show that the total loaded and deterministic empty trip distances for the loaded trip optimal design is 11210 . However, this number reduces to 7670 under our optimal design. The ratio of the total loaded and deterministic empty trip distances in loaded trip optimal design to that of our optimal design is 1.46. The following simulation study shows that ratio of loaded and stochastic trips in the simulation model to loaded and deterministic empty trips in our optimal design will never reach 1.46. (Obviously the ratio of loaded and stochastic trips to loaded and deterministic empty trips under loaded trip minimization model is always greater than 1.46.)

The test bed of this simulation study is the 11 cell prototype problem used by Sinriech and Tanchoco (1993). We examine a large set of scenarios to measure the following responses

Sim/Opt: The ratio of the total loaded and stochastic empty trips in the simulation model to the loaded and deterministic empty trips in the optimization model.

Qin: The average incoming queue at each manufacturing cell.

Qout: The average outgoing queue at each manufacturing cell.

Ws/Wr: The ratio of the average waiting time in the system to that of the waiting time in the servers. The waiting time in the servers is the pure manufacturing time. The waiting time in the system includes the waiting time in the 
servers, the waiting time in queues, and the handling time on AGVs.

$\mathrm{Sim} / \mathrm{Opt}$ is the primary response to show that the total trip distances (or the number of rotations around the loop) in the simulation environment is closely estimated by the optimization model. It is accompanied by the other responses to show that this performance is not achieved at the expense of a long flow time or a large inventory.

The responses are tested against the changes in combinations of the following factors:

IMF: Intensity of material flow. The material flow chart of Figure 1 was prepared based on the following information (Sinriech and Tanchoco, 1993). Product mix is 5, 3, 1, 2 , and 5 unit loads for product 1 to product 5, respectively. A total of 80 unit loads are completed in 8 working hours. Production routings are; Product 1: 1-2-4-9-8-10-11, Product 2: 1-2-4-7-9-4-6-10-11, Product 3: 1-2-7-9-6-10-11, Product 4: 1-2-3-5-9-6-11, and Product 5: 1-2-4-8-10-11. In order to increase the required number of vehicles, we will examine two other material flow matrices, where their elements are two and three times the elements of the base loaded flow chart of Figure 1(b).

P/D time: Pickup and dropoff times. Since congestion has been modeled in none of the models developed to design flow path or station locations, an assumption of zero pickup or dropoff times does not invalidate the results. However, we examine zero P/D times, as well as $15 \mathrm{sec}-$ onds of pickup or dropoff time, respectively

UMC: Utilization of manufacturing cells. The utilization of the manufacturing machinery is set to $0.6,0.75$, and 0.9 .

ECV: Erlang coefficient of variation of manufacturing processing times.

The processing time of the manufacturing operations follows Erlang distribution with coefficient of variation of 4 , 2 , and 1 .

The simulation results represented in Table 1 are promising. The loaded and stochastic empty trips in the simulation model developed based on our optimal design, will never reach the equivalent loaded and deterministic empty trips obtained under the previous optimal designs for this problem. The number of rotations in the simulation model in the worst case is $20 \backslash \%$ above the value of the objective function of our deterministic optimal design.

We have already shown that the optimal solution obtained under the loaded trip distance optimal design is $46 \backslash \%$ away from the actual loaded and deterministic empty trip distances obtained in our optimal design. Obviously, the total loaded and stochastic empty trip distances of the loaded trip minimal design will show a larger gap.
In summary, the total loaded and stochastic empty trips are closely estimated by the objective function in our optimization model. The minimization of loaded and deterministic empty vehicle trips under STDF dispatching policy in the design phase seems an effective way to minimize the stochastic empty trips in the operation phase.

\section{CONCLUSION}

This paper considers the design of an optimal material flow path loop between fixed manufacturing cells served by unit load AGVs. Previous studies of this problem have considered only loaded vehicle travel. While the importance of empty trips are know well known, a majority of literature recommend FCFS dispatching. FCFS not only incorporates empty trips it also legitimize earlier loaded trip optimization models by stating that empty trips can also addressed as loaded trips. A primary interest of our work was to illustrate the superiority of the STDF over the FCFS when integrated into the design phase.

We also showed that optimization under STDF in the design phase produces the best solution for FEFS in the operation phase. We now recommend to design the loop and station locations using STDF dispatching, and to operate on this material handling network follow decentralized FEFS dispatching.

Table 1: The ratio of the total trip distances in the simulation model to that of the optimization model under different simulation model parameters.

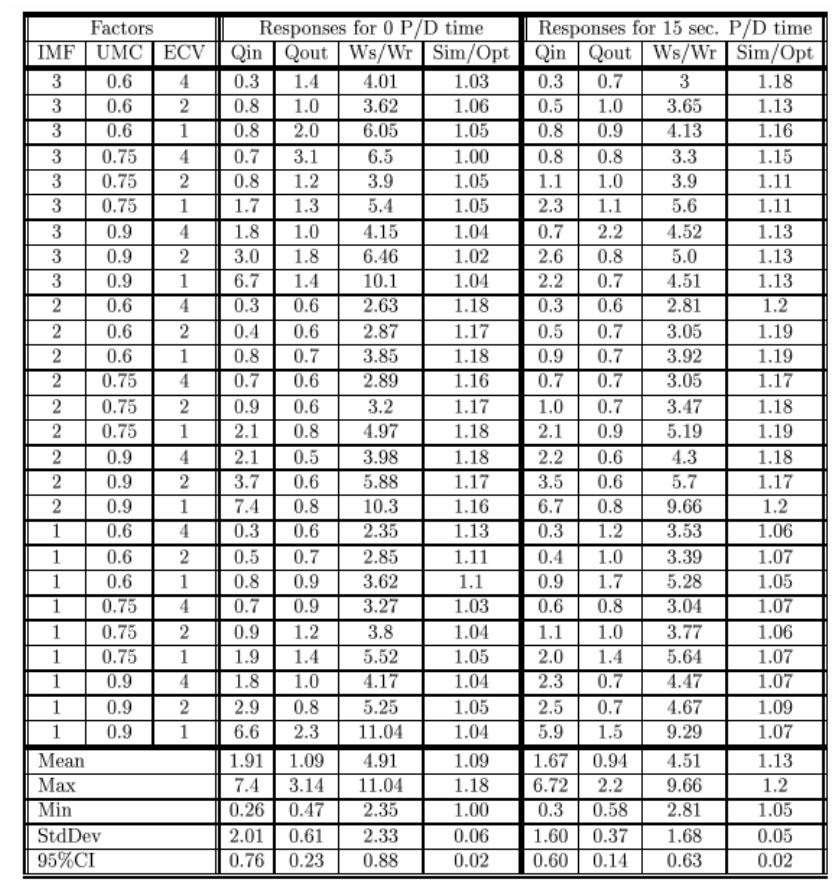




\section{REFERENCES}

Sinriech, D. and J.M.A. Tanchoco. 1993. Solution methods for the mathematical models of single-loop AGV systems. International Journal of Production Research. 31:705-725.

\section{AUTHOR BIOGRAPHIES}

Ardavan Asef-Vaziri is an assistant professor at the department of Systems and Operations Management, College of Business Administration and Economics, California State University, Northridge. His research interests revolve around applications of integer programming and simulation in facility logistics and lean business process flow design. 